\title{
Comparative genomics on mercury methylators (Pseudo)Desulfovibrio strains
}

MARISOL GOÑI URRIZA ${ }^{1}$, BAHIA KHALFAOUI-HASSANI ${ }^{1}$, MATHILDE MONPERRUS ${ }^{2}$, REMY GUYONEAUD ${ }^{1}$

${ }^{1}$ Universite de Pau et des Pays de l'Adour, E2S UPPA, CNRS, IPREM, Pau, France. marisol.goni@univ-pau.fr

${ }^{2}$ Universite de Pau et des Pays de l'Adour, E2S UPPA, CNRS,

IPREM, Anglet, France. mathilde.monperrus@univ-pau.fr

Mercury methylation is performed by anaerobic microorganisms including several strains related to Pseudodesulfovibrio and Desulfovibrio genera. The consequence of inorganic mercury transformation to methylmercury can be worrisome for human health and the environment. In order to provide new insights into the molecular mechanisms of mercury methylation, we performed a comparative genomic analysis on mercury methylators and non-methylators from (Pseudo)Desulfovibrio strains. Our results showed that (Pseudo)Desulfovibrio species are phylogenetically and metabolically distant and that strains able to perform methylation are affiliated with one branch of the phylogenetic tree. However, except for $h g c A$ and $h g c B$ genes, no other specific genetic markers were found among methylating strains. $h g c A$ and $h g c B$ genes can be found adjacent or separated, but proximity between those genes does not promote higher mercury methylation. In addition, close examination of the nonmethylator Pseudodesulfovibrio piezophilus C1TLV30 strain, showed a syntenic structure that suggests a recombination event and may have led to $h g c B$ depletion. The genomic analyses identify also ars $R$ gene coding for a putative regulator upstream $h g c A$. Both genes are cotranscribed suggesting a role of ArsR in $h g c A$ expression and probably a role in mercury methylation. 